\title{
THE RELATIONSHIP BETWEEN THE STRUCTURAL AND ORTHOGONAL COMPRESSIVE PROPERTIES OF TRABECULAR BONE
}

\author{
R. W. Goulet, ${ }^{*}$ S. A. Goldstein, ${ }^{*}$ M. J. Ciarelli, ${ }^{*}$ J. L. Kuhn, ${ }^{*}$ M. B. Brown $\dagger$ and \\ L. A. FELDKAMP $\ddagger$ \\ *Orthopaedic Research Laboratories, Section of Orthopaedic Surgery, The University of Michigan, \\ Ann Arbor, MI 48109, U.S.A.; +Department of Biostatistics, School of Public Health, The University \\ of Michigan, Ann Arbor, MI 48109, U.S.A.; and $\ddagger$ Research Laboratory, Ford Motor Company, \\ Dearborn, MI 48121, U.S.A.
}

\begin{abstract}
In this study, cubes of trabecular bone with a wide range of structural properties were scanned on a micro-computed tomography system to produce complete three-dimensional digitizations from which morphological and architectural parameters could be measured in a nondestructive manner. The cubes were then mechanically tested in uniaxial compression in three orthogonal directions and to failure in one direction to find the orthogonal tangent elastic moduli and ultimate strengths. After testing, the cubes were weighed and ashed to determine the apparent and ash densities. A high correlation between the basic stereologic measurements was found, indicating that there is a relationship between the amount of bone and number of trabeculae in cancellous bone. Regression analysis was used to estimate the modulus and ultimate strength; these regressions accounted for $68-90 \%$ of the variance in these measures. These relationships were dependent on the metaphyseal type and donor, with the modulus also dependent on the direction of testing. This indicates that the properties of the individual trabeculae, as well as their amount and organization, may be important in predicting the mechanical propertics of cancellous bone.
\end{abstract}

\section{INTRODUCTION}

The structural and mechanical characteristics of cancellous bone have been studied by many investigators to understand better the functional adaptation associated with the wide range of properties documented for this biological material. It is known that morphology varies between bones and even within a bone (Pugh et al., 1973; Raux et al., 1975; Singh, 1978; Townsend et al., 1975; Williams and Lewis, 1982; Whitehouse et al., 1971) as do the mechanical properties (Brown and Ferguson, 1980; Ciarelli et al., 1991; Goldstein, 1987). The density of cancellous bone has been related to its strength and modulus (Carter and Hayes, 1977; Ciarelli et al., 1991; Gibson, 1985; Hvid et al., 1985; Rice et al., 1988). While measures of density often lead to reasonable estimates of mechanical properties, they do not explain all the variance nor do they account for the structural organization of the trabecular bone, thus rendering a scalar measure inadequate for predicting the material properties.

Whitehouse (1974) was able to describe the structural anisotropy of trabecular bone on planar sections. Harrigan and Mann (1984) expanded this planar analysis to three dimensions, producing a second rank symmetric tensor to describe the anisotropy, while imposing the assumption of at least orthotropic symmetry. Cowin (1985) was able to

Received in final form 9 September 1993.

Author to whom correspondence should be addressed: Steven A. Goldstein, Orthopaedic Research Laboratories, University of Michigan, G-0161 400 N. Ingalls, Ann Arbor, MI 48109-0486, U.S.A. relate this tensor, in the form of a fabric tensor, to the compliance matrix. This leads to the ability to relate the structural organization of trabecular bone to the mechanical properties in a mathematical equation and the possibility of developing analytical models for the prediction of trabecular bone remodeling and its relationship to the stress environment (Cowin, 1986). Turner et al. (1990) have recently presented results using this fabric tensor to predict the mechanical properties of cancellous bone from bovine femora and human tibias.

Improved quantification of the structural organization of trabecular bone permits more comprehensive assessment of the intricate relationship between trabecular bone and its environment. One can then look more rigorously at schemes or strategies the body might use to optimize its structure while maintaining structural integrity and strength. The effects of various pathological disorders on bone can also be studied and treatments designed to specifically maintain the most important structural parameters for adequate bone strength could be advanced. These relationships may also assist in estimating material properties for finite element analyses that investigate trabecular bone adaptation.

The purpose of this study is to investigate the relationships between the structural parameters for cancellous bone, to determine their correlation to the mechanical properties, and to evaluate which parameters are important for maintaining bone strength and integrity. These relationships for normal individuals provide insight into the complex interactions of the architecture of the trabecular bone. 


\section{METHODS AND MATERIALS}

Human cancellous bone specimens from seven metaphyseal regions of four human cadavers were used: proximal tibia, proximal and distal femora, iliac crest, distal radius, proximal humerus, and the lumber vertebral bodies. There were two females, aged 55 and 61 , and two males, aged 31 and 69 , with no known bone disorders. The metaphyseal region plus a portion of the diaphysis was cut from each long bone. The diaphyseal portions were embedded in square aluminium blocks using Vel-Mix Stone (Kerr Co., Chicago, IL) so that cubes of metaphyseal cancellous bone could be cut along the anatomical axes of anterior-posterior (AP), medial-lateral (ML), and inferior-superior (IS). The iliac crest and vertebral bodies were mounted to acrylic blocks using bone cement in an orientation that also allowed cubes to be cut along the anatomical axes. The blocks were then mounted to the $x-y$ table of a numerically controlled milling machine (Model Series I CNC, Bridgeport Machines Inc., Troy, MI 48084). A stainless steel blade rotating at low speed under constant water irrigation was used to cut the specimens. Articular surfaces were removed to expose a first layer of trabecular bone in a plane perpendicular to an anatomical axis of the bone. Eight millimeter trabecular bone cubes were then produced from successive layers. The location and orientation of each bone cube were carefully documented and only those cubes composed entirely of cancellous bone were included in the study. The cubes were stored in lactated Ringers solution and kept frozen at $-20^{\circ} \mathrm{C}$ until scanning and mechanical testing. To ensure a wide range of morphological and structural properties, at least seven specimens spread over the whole metaphyseal region of the larger bones were used. From the smaller metaphyseal regions, only 2-4 specimens were produced and all were used in the study. Table 1 shows the distribution of the 104 specimens by donor and metaphyseal type used for scanning and mechanical testing.

To generate a complete three-dimensional digitized image of each specimen (Fig. 1) for the stereologic analysis, specimens were scanned on a unique micro-

Table 1. Distribution of trabecular cube locations from cadaveric specimens

\begin{tabular}{lrrrrrr}
\hline & \multicolumn{3}{c}{ Male } & \multicolumn{2}{c}{ Female } & \\
\cline { 2 - 3 } Ages & \multicolumn{1}{c}{31} & 69 & 55 & 61 & Total \\
\hline Proximal tibia & 5 & 10 & 10 & 2 & 27 \\
Distal femur & 10 & 10 & 10 & 9 & 39 \\
Proximal femur & 5 & 2 & 2 & 3 & 12 \\
Iliac crest & - & 1 & 3 & - & 4 \\
Spine & - & - & 4 & - & 4 \\
Distal radius & 3 & 2 & 2 & 2 & 9 \\
Proximal humerus & 3 & 2 & 2 & 2 & 9 \\
Total & 26 & 27 & 33 & 18 & 104 \\
\hline
\end{tabular}

computed tomography (micro-CT) system (Feldkamp et al., 1984, 1989) at the Ford Motor Company Research Laboratories, Dearborn, MI. The specimens (partially thawed and removed from Ringers solution) were mounted on a special holder in air for scanning which took approximately $15-20 \mathrm{~min}$ for each cube. All cubes were reconstructed on a $50 \mu \mathrm{m}$ mesh, so that each data point of the reconstruction represented a subvolume of the actual specimen of $\left(50 \times 50 \times 50 \mu \mathrm{m}^{3}\right)$. The magnification for scanning was approximately $0.045 \mathrm{~mm}_{\text {pixel }}{ }^{-1}$ giving a resolution between $50-70 \mu \mathrm{m}$. The data size of the reconstruction consisted of approximately $181 \times 181 \times 125$ data points, with each data point converted to one byte having a grayscale value between 0 and 255 . The reconstructed images were stored on magnetic tape until the stereologic analyses were performed on an Apollo Domain Computing system (DN4000). After scanning, the specimens were returned to Ringers solution and refrozen until mechanical testing.

For the mechanical testing procedure, the specimens were thawed and tested at room temperature in uniaxial compression at a displacement rate of $0.01 \mathrm{~s}^{-1}$ in each of the three orthogonal directions (AP, ML, IS) to pre-yield to find the tangent moduli, and to failure in one random direction to obtain the ultimate strength. The testing was done on an Instron Materials Testing machine (Model 1000, Instron Corp., Canton, MA 02021), with a Zenith-286 PC equipped with a Data Translation A/D board for data collection and analysis. The protocol for testing consisted of preconditioning the cube by cyclic loading 10 times to approximately $50-60 \%$ of the estimated ultimate strength as recommended by the work of Linde and Hvid (1987), and then the next cycle was used for data collection. $A$ priori estimates for the ultimate strengths were based on previous experiments (Ciarelli et al., 1991). An oscilloscope was used to monitor the load vs displacement curves during the preconditioning and final test cycles to ensure that the loads remained within the elastic region. The loads were converted to stress values by dividing by the original cross-sectional area of the specimen perpendicular to the direction of testing. Displacement was measured from the cross-head motion and the tangent modulus (MOD) was calculated from the most linear portion of the stress-strain curve with a correction applied to account for the system compliance. This testing procedure was carried out in all three directions with the last direction carried out to failure to determine the ultimate strength $\left(S_{u}\right)$ for one direction of the specimen. The ultimate strength was defined as the maximum stress level achieved during the failure test.

Once mechanically tested, the apparent and ash densities were detcrmined based on a protocol described in Ciarelli et al. (1991). The marrow from the specimens was removed using a water-air jet, then centrifuged to remove all excess water and weighed to determine the apparent density. They were then ashed 


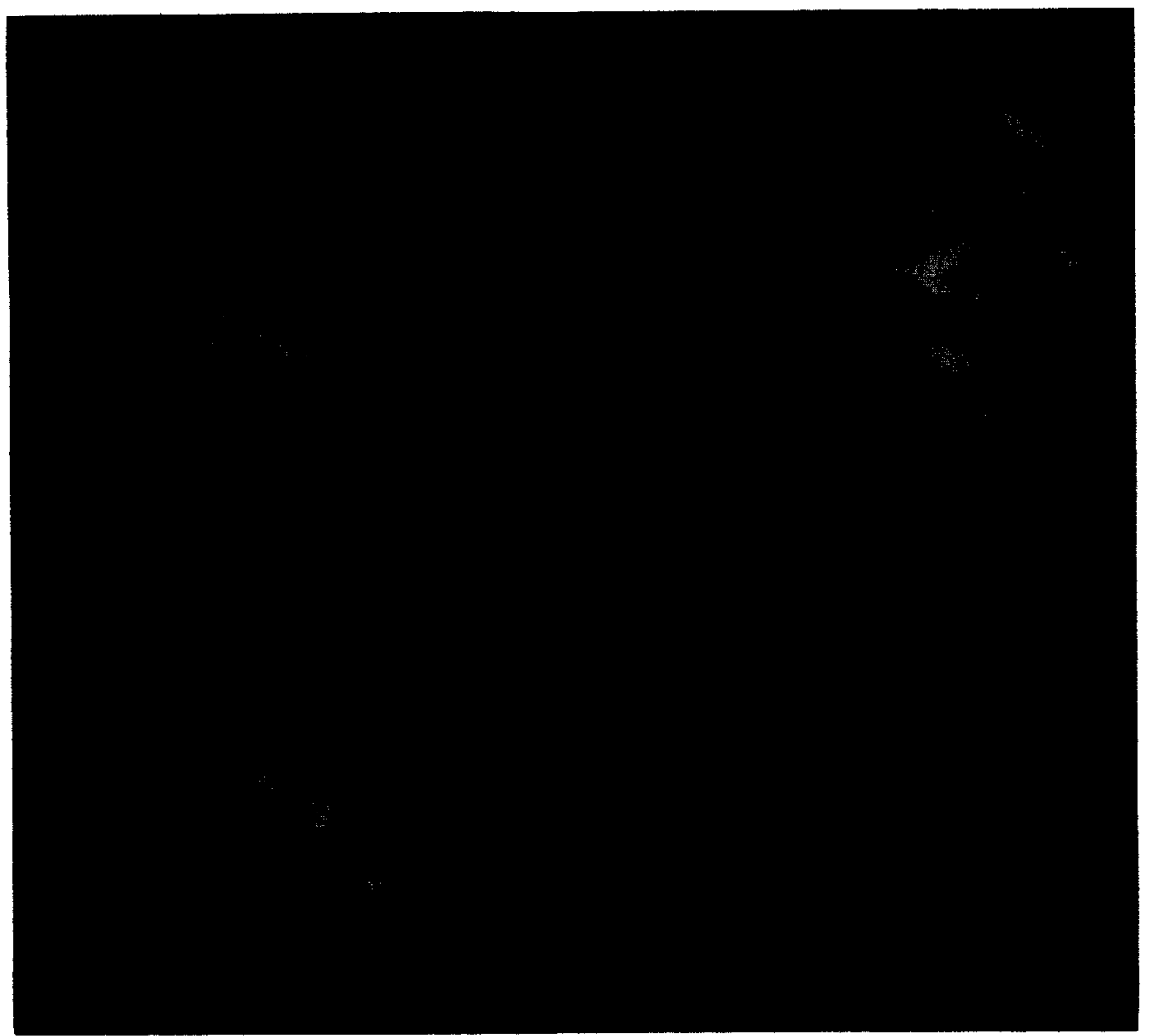

Fig. 1. Micro-CT image of a trabecular bone cube showing the full three-dimensional image and orthogonal two-dimensional slices of the specimen. The image was reconstructed on a $50 \mu \mathrm{m}$ mesh and thresholded. The $x$-direction corresponds to the anterior-posterior direction, $y$-direction to medial-lateral, and $z$-direction to inferior-superior. 
and reweighed to determine the ash density. The volume of the specimen was determined from micrometer measurements of the specimen's dimensions.

The morphological analyses consisted of first applying an adaptive thresholding procedure (Feldkamp et al., 1989; Kuhn et al., 1990) to each reconstructed micro-CT image to separate the image into bone/nonbone voxels. Because the X-ray attenuations through a nonhomogeneous material are not uniform and there may exist trabeculae of varying densities throughout the specimen, the selection of one grayscaled threshold value to create a binary image was inappropriate. Therefore, a thresholding procedure was developed to account for the possible variation in density levels of the trabeculae throughout the specimen. This thresholding procedure has been evaluated by a comparison of micro-CT image slices within bone cubes and matching histological sections (Kuhn et al., 1990). Errors of less than 4 and $15 \%$ were found for the two basic stereologic measurements used to derive the morphological and architectural parameters: $P_{\mathbf{P}}$, the number of bone voxels per total number of voxels in an analysis region and $P_{\mathrm{L}}$, the number of intersections between bone and nonbone components per total length of test lines applied to a specimen. This error in $P_{\mathrm{L}}$ amounts to about $19 \mu \mathrm{m}$ for the length variables, i.e., trabecular plate thickness.

The equations for the morphology parameters of interest as described by Underwood (1970), Weibel (1979), Parfitt et al. (1983), and others are (the nomenclature recommended for use in bone histomorphometry will be used, Parfitt et al., 1987): ratio of bone, $\mathrm{BS} / \mathrm{BV}$, is a measure of the surface of trabecular bone (BS) to the volume of trabecular bone with units of $\mathrm{mm}^{2} \mathrm{~mm}^{-3}$. The trabecular plate thickness, Tb.Th, trabecular plate number, Tb.N, and trabecular plate separation, $\mathrm{Tb} . \mathrm{Sp}$, all assume a plate model for the trabecular bone in the derivation of the equations used to calculate these quantities. Trabecular plate thickness is a measure of the average thickness of the trabeculae within the whole specimen and has units of $\mathrm{mm}$. Trabecular plate number is a measure of how many trabeculae there are in a specimen and has units of $\mathrm{mm}^{-1}$. Trabecular plate separation is a measure of the average distance between trabecular plates and has units of $\mathrm{mm}$.

The topological measure of connectivity, or Euler-Poincaré number, was measured based on a procedure described in general terms by Serra (1982) and in detail by Feldkamp et al. (1989). The Euler number is multiplied by negative one to produce a quantity which effectively counts the number of connections in a specimen. The number is then normalized by dividing by the volume analyzed to allow meaningful comparison between specimens of different volumes (this variable will be denoted as $-\mathrm{Eu}$ ler/Vol). Even though this variable gives a measure of how connected the material is, it provides no information about the quality of the connections. A limitation of the Euler-Poincare number as determined here is that it does not take into account trabeculae at the edge of the analysis volume that are not connected
Bone volume fraction $\left(\mathrm{mm}^{3} \mathrm{~mm}^{-3}\right)$

Trabecular plate number $\left(\mathrm{mm}^{-1}\right)$

Trabecular plate thickness $(\mathrm{mm})$

Trabecular plate separation $(\mathrm{mm})$

Surface-to-volume ratio of bone $\left(\mathrm{mm}^{2} \mathrm{~mm}^{-3}\right)$

$$
\begin{aligned}
& \text { BV } / \mathrm{TV}=P_{\mathrm{P}}, \\
& \text { Tb.N }=P_{\mathrm{L}}, \\
& \text { Tb.Th }=P_{\mathrm{P}} / P_{\mathrm{L}}, \\
& \text { Tb.Sp }=\left(1-P_{\mathrm{P}}\right) / P_{\mathrm{L}}, \\
& \mathrm{BS} / \mathrm{BV}=2 * P_{\mathrm{L}} / P_{\mathrm{P}},
\end{aligned}
$$

where each voxel is used to find $P_{\mathrm{P}}$, and perpendicular test lines superimposed over the micro-CT image are used to find $P_{\mathbf{L}}$. Because of the anisotropic nature of cancellous bone, a single set of parallel test lines superimposed over the image should not be used to find $P_{\mathbf{L}}$. The use of perpendicular test lines and a calculation of an average $P_{\mathrm{L}}$ from the three orientations gives a reasonable estimate of the true $P_{\mathbf{L}}$ (Weibel, 1980). Further, in our procedure the whole specimen is analyzed, rather than one random plane from the specimen; our procedure should give a better estimate of the true $P_{\mathrm{P}}$ and $P_{\mathrm{L}}$. Turner (1986) calculated an average $P_{L}$ as a function of the principal mean intercept lengths. A small substudy of our data showed that this produces an estimate that was highly correlated to an average $P_{\mathrm{L}}$ determined from orthogonal test lines; slope $=1.00, R^{2}=0.99$.

The bone volume fraction, BV/TV, is a measure of the volume of trabecular bone (BV) per total volume (TV) of analysis in the cube and is expressed as a fraction with units of $\mathrm{mm}^{3} \mathrm{~mm}^{-3}$. The surface to volume due to being disconnected by the analysis volume; or trabeculae that appear to be free floating within the analysis volume due to the limitations of the scanning and thresholding procedures. These errors will lead to a slightly more positive Euler number (negative as used in this paper), but we believe the magnitude of the correlations seen will remain the same. A subset of 21 specimens were analyzed to study this problem by removing single voxels and disconnected trabeculae so that only the most continuous structure would be analyzed.

The quantification of the anisotropy was determined by a procedure described by Whitehouse (1974) for calculating the mean intercept length of trabecular bone, denoted as MIL, on a two-dimensional analysis section. A circular analysis region was defined on each micro-CT slice. The areal density, $P_{\mathrm{P}}$, of the analysis region was found by dividing the total number of bone pixels by the total number of pixels in the analysis region. An array of parallel test lines was then 
superimposed over the specimen in this analysis region at an angle, $\Theta$, from a reference direction. The number of intersections between the bone and nonbone components was counted for each test line. The total number of intersections for all test lines was divided by the total length of test lines to give the number of intersections per unit length of test line, $P_{\mathrm{L}}(\Theta)$. The mean intercept length for trabecular bone was calculated as

$$
\operatorname{MIL}(\Theta)=2 * \frac{P_{\mathrm{P}}}{P_{\mathrm{L}}(\Theta)} \quad \text { (units in } \mathrm{mm} \text { ). }
$$

The grid was then rotated, $\Delta \Theta$ (a $5^{\circ}$ increment was used), and the same calculations repeated. This gave $\operatorname{MIL}(\Theta)$ as a function of the grid angle orientation over the image, where $0 \leqslant \Theta<180^{\circ}$. Plotting $\operatorname{MIL}(\Theta)$ vs $\Theta$ on a polar plot generally produces an ellipticalshaped curve. The data were fitted to the equation of an ellipse from which the direction and amount of orientation could be found for that analysis plane.

By analyzing three orthogonal planes with this method, the three-dimensional anisotropy of the specimen may be quantified as shown by Harrigan and Mann (1984). This method assumes orthotropic symmetry and that the anisotropy may be described by an ellipsoid. The equation of an ellipsoid may be calculated if it is assumed that the ellipses determined from the analysis planes are the projections of the ellipsoid on them. The general form of the equation is

$$
\frac{1}{\operatorname{MIL}^{2}(\mathbf{n})}=\{\mathbf{n}\}^{\mathrm{T}}[\mathbf{A}]\{\mathbf{n}\},
$$

where $\mathbf{n}$ is the unit vector in the direction of interest, and [A] is the second rank symmetric anisotropy tensor formed from the coefficients of the equation of the elipsoid. By solving the eigenvalue problem for this tensor, the principal structural directions and magnitudes are obtained. The eigenvalues are used to define the degree of anisotropy, DA, as the maximum to minimum MIL ratio:

$$
\mathrm{DA}=\frac{\mathrm{MIL}_{1}}{\mathrm{MiLL}_{3}}=\sqrt{\frac{\lambda_{3}}{\lambda_{1}}},
$$

with $\mathrm{MIL}_{i}=1 / \sqrt{\lambda_{i}}$, where $\lambda_{i}$ are the eigenvalues of [A], and $\mathrm{MIL}_{1}>\mathrm{MIL}_{2}>\mathrm{MIL}_{3}$. In this study, a single subscript will refer to the principal structural directions, while a double subscript will refer to the axes of the specimen: $11=\mathrm{AP}, 22=\mathrm{ML}$, and $33=\mathrm{IS}$. Taking the inverse square root of the anisotropy tensor, [A], will result in what Cowin (1985) has called the Fabric tensor, $[\mathbf{H}]$, for cancellous bone. This fabric tensor would then be normalized by dividing by the trace of the tensor. In our analysis, we have used the un-normalized mean intercept length values for the formulation of an empirical structure-function relationship.

Because of the nature of the three-dimensional digitization of the cube, we were able to analyze any three orthogonal planes within the specimen along with the surface planes. The anisotropy tensor has been formed using three surface orthogonal analysis planes, three central orthogonal analysis planes, or three orthogonal planes that represent the average ellipse in each of the three orthogonal directions. This average plane ellipse was found from analyzing the cube in a front-to-back procedure and calculating the average $\operatorname{MIL}(\Theta)$ from all planes for each grid angle orientation. These average $\operatorname{MIL}(\Theta)$ were then used to form the two-dimensional ellipse for that direction. The analyses will be referred to as surface, central, and average throughout the rest of the text. For this study, only the anisotropy tensor formed from the average plane analysis will be used in the derivation of the structure-function relationship. The average plane analysis will be used since more of the three-dimensional image is analyzed to produce the tensor that gives a more accurate representation of the anisotropy (Goulet et al., 1989).

For the statistical analyses, Pearson product correlations were used to study the associations between the various morphological and architectural parameters. Linear regressions were used to model the relationships between the morphological and architectural parameters on the observed scale. Linear regression models were fit to the logarithms (base 10) of the three moduli and ultimate strength with the independent variables being the morphology and architectural measures, either on the original scale or after a logarithmic transformation. The average of the $\log \left(\mathrm{MOD}_{\mathrm{ii}}\right)$, calculated as $\left\{\left[\log \left(\mathrm{MOD}_{11}\right)+\log \left(\mathrm{MOD}_{22}\right)+\log \left(\mathrm{MOD}_{33}\right)\right] / 3\right\}$ and denoted as Ave: $\log (M O D)$, and the average of $\log \left(\mathrm{MIL}_{\mathrm{ii}}\right)$, calculated as $\left\{\left[\log \left(\mathrm{MIL}_{11}\right)+\log \left(\mathrm{MIL}_{22}\right)\right.\right.$ $\left.\left.+\log \left(\mathrm{MIL}_{\mathbf{3 3}_{3}}\right)\right] / 3\right\}$ and denoted as Ave: $\log (\mathrm{MIL})$, were also determined for each cube. The standard error of the estimate (SEE) and p-values greater than 0.005 were presented with each regression. All regressions were based on 104 observations unless noted otherwise. A set of dummy variables was created to represent a categorical variable defining the direction of testing, and included in the models to test for the independence of mechanical testing direction. Sets of dummy variables were also created for the categorical variables representing the subject (donor) and the type of bone specimen (metaphyseal type). The use of these dummy variables removes donor-to-donor differences or bone-to-bone differences from the estimate of the correlations and from the estimates of the intercepts of the regression equations. The statistical analyses were performed using SYSTAT and BMDP statistical computer packages.

\section{RESULTS}

The descriptive statistics of the architectural and mechanical parameters are presented in Table 2 . The specimens included in this study ranged in bone volume fraction from 6 to $36 \%$ and had a degree of anisotropy from 1.11 to 2.54 (from the average 
Table 2. Basic descriptive statistics of the data

\begin{tabular}{lrrrrr}
\hline Variable & $n$ & Mean & S.D. & Median & Range \\
\hline BV/TV $\left(\mathrm{mm}^{3} \mathrm{~mm}^{-3}\right)$ & 104 & 0.20 & 0.07 & 0.20 & $0.06-0.36$ \\
Tb.N (mm $\left.{ }^{-1}\right)$ & 104 & 1.39 & 0.32 & 1.37 & $0.61-2.06$ \\
Tb.Th (mm) & 104 & 0.14 & 0.02 & 0.15 & $0.10-0.19$ \\
Tb.Sp (mm) & 104 & 0.64 & 0.24 & 0.61 & $0.32-1.67$ \\
BS/BV (mm $\left.{ }^{2} \mathrm{~mm}^{-3}\right)$ & 104 & 14.44 & 2.21 & 13.93 & $10.75-21.02$ \\
- Euler/Vol (mm $\left.{ }^{-3}\right)$ & 104 & 2.83 & 1.88 & 2.78 & $-1.63-6.56$ \\
DA (mm mm $\left.{ }^{-1}\right)$ & 104 & 1.65 & 0.29 & 1.62 & $1.11-2.54$ \\
AP MIL (mm) & 104 & 0.30 & 0.06 & 0.29 & $0.21-0.47$ \\
ML MIL (mm) & 104 & 0.26 & 0.04 & 0.26 & $0.19-0.41$ \\
IS MIL (mm) & 104 & 0.36 & 0.06 & 0.35 & $0.24-0.55$ \\
Apparent density & 104 & 0.31 & 0.12 & 0.30 & $0.11-0.60$ \\
Ash density & 104 & 0.19 & 0.07 & 0.18 & $0.07-0.37$ \\
AP modulus (MPa) & 104 & 173 & 204 & 120 & $6-1524$ \\
ML modulus (MPa) & 104 & 123 & 120 & 89 & $1-654$ \\
IS modulus (MPa) & 104 & 287 & 255 & 208 & $16-1113$ \\
AP Uit. strength (MPa) & 32 & 3.31 & 3.14 & 2.90 & $0.58-16.00$ \\
ML Ult. strength (MPa) & 34 & 2.58 & 2.19 & 1.88 & $0.10-9.60$ \\
IS Ult. strength (MPa) & 38 & 4.63 & 3.48 & 3.78 & $0.51-14.56$ \\
\hline
\end{tabular}

Table 3. Pearson product moment correlations between the various morphological and architectural parameters

\begin{tabular}{|c|c|c|c|c|c|c|c|c|c|}
\hline & $\mathrm{BV} / \mathrm{TV}$ & Tb.N & -Euler/Vol & DA & BS/BV & Tb.Th & Tb.Sp & Ash Den & App Den \\
\hline $\mathrm{BV} / \mathrm{TV}$ & 1.000 & & & & & & & & \\
\hline Tb.N & 0.955 & 1.000 & & & & & & & \\
\hline - Euler/Vol & 0.874 & 0.952 & 1.000 & & & & & & \\
\hline D.A. & 0.281 & 0.265 & 0.123 & 1.000 & & & & & \\
\hline $\mathrm{BS} / \mathrm{BV}$ & -0.863 & -0.731 & -0.631 & -0.263 & 1.000 & & & & \\
\hline Tb.Th & 0.872 & 0.710 & 0.595 & 0.301 & -0.983 & 1.000 & & & \\
\hline Tb.Sp & -0.892 & -0.934 & -0.880 & -0.243 & 0.801 & -0.742 & 1.000 & & \\
\hline Ash Den & 0.937 & 0.875 & 0.795 & 0.205 & -0.797 & 0.822 & -0.786 & 1.000 & \\
\hline App Den & 0.903 & 0.832 & 0.751 & 0.187 & -0.782 & 0.809 & -0.757 & 0.969 & 1.000 \\
\hline
\end{tabular}

Correlations of 0.195 and 0.254 represent critical values corresponding to two-tailed 5 and $1 \%$ levels of significance. All measures are significantly correlated at $p=0.001$ level except degree of anisotropy (DA).

plane analysis). The thickness of the trabeculae varied from 0.10 to $0.19 \mathrm{~mm}$ and had a separation of $0.32-1.67 \mathrm{~mm}$. Frequency distribution plots of some of the various morphological and architectural measures are shown in Fig. 2(a)-(d). The mechanical properties exhibited skewed distribution [Fig. 2(e) and (f)].

Investigating the relationship between the various structural properties, correlations between the morphology variables and connectivity were highly significant, $p<0.001$, except when correlated with the degree of anisotropy, DA (Table 3). The most interesting of these correlations were the relationships between connectivity ( - Euler/Vol), trabecular plate number (Tb.N) and bone volume fraction (BV/TV). All three of these measures were derived with different stereological procedures and are not related mathematically, yet were highly correlated, $r=0.95$ for - Euler/Vol vs Tb.N, $r=0.87$ for - Euler/Vol vs BV/TV, and $r=0.96$ for Tb.N vs BV/TV [Table 3 and Fig. 3(a)-(c)]. The other morphological variables (Tb.Th, Tb.Sp, and BS/BV) were derived using the measurements BV/TV and Tb.N [equations (1)-(5)].
The relationships between these variables are mathematically dependent on how the basic measurements were combined to compute them and thus are not shown. The degree of anisotropy, DA, was less correlated with the other variables, although all correlations are significant $(p<0.05)$. When partial correlations are computed for the variables found in Table 3 after adjusting for donor and metaphyseal bone type, all large correlations remained large $(p<0.001)$; the correlation of DA with - Euler/Vol is no longer significant $(r=0.09)$, all other correlations with DA remain significant $(p<0.05)$.

Because of the limitations of the scanning and thresholding procedures, there is the possibility that single voxels or disconnected trabeculae may be present in the image. To test the affect of this error on the correlations between BV/TV, Tb.N, and - Euler/Vol, a subset of specimen $(n=21)$ were used. After removing single voxels and disconnected trabeculae, it was found that the magnitude of the correlations between - Euler/Vol, Tb.N and BV/TV remained the same $(r=0.93$ for - Euler/Vol vs Tb.N, $r=0.88$ for $-\mathrm{Eu}$ ler/Vol vs BV/TV, and $r=0.97$ for Tb.N vs BV/TV) as 
(a)

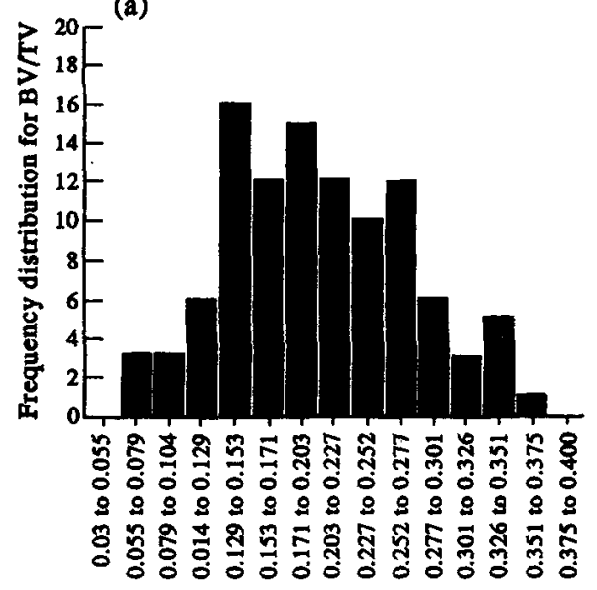

(Units are $\mathrm{mm}^{3} / \mathrm{mm}^{3}$ )

(c)

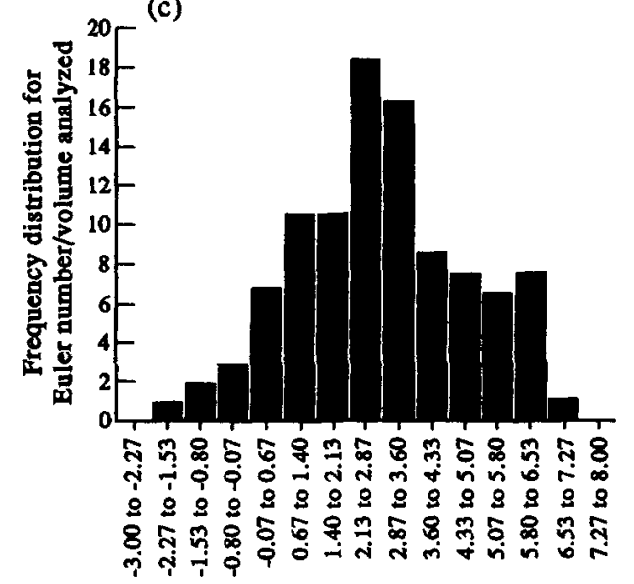

(Units are $1 / \mathrm{mm}^{3}$ )

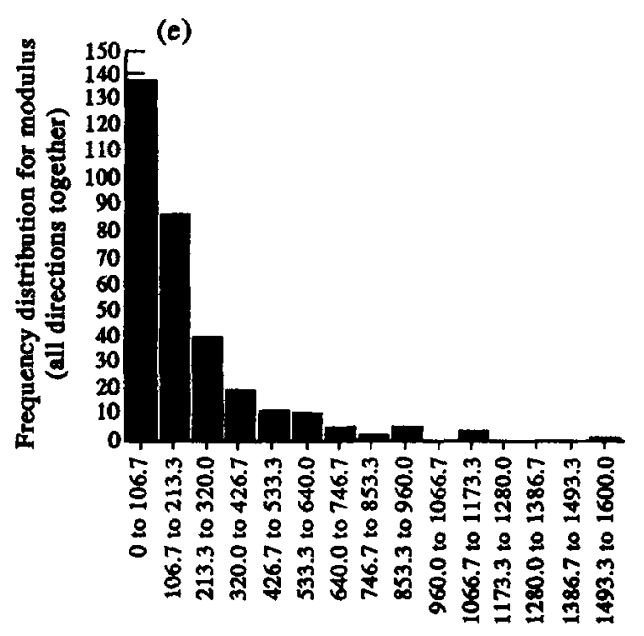

(Units are MPa) (b)

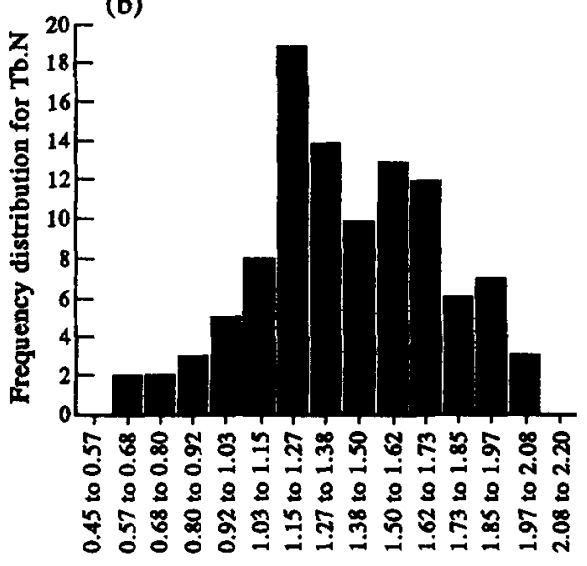

(Units are 1/mm)

(d)

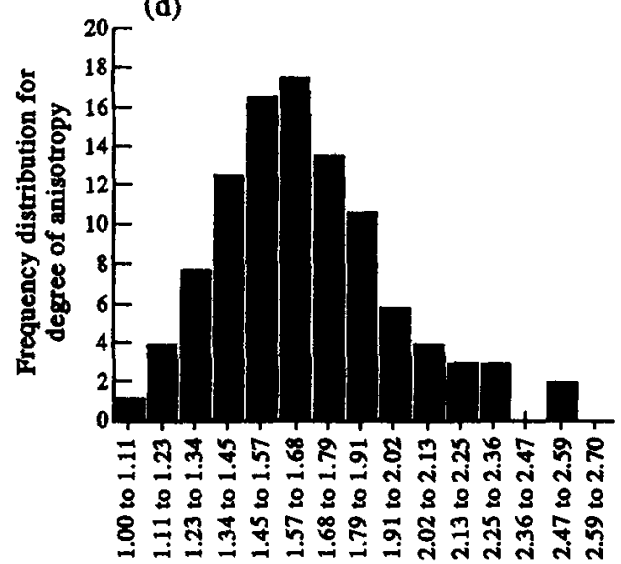

(Dimensionless)

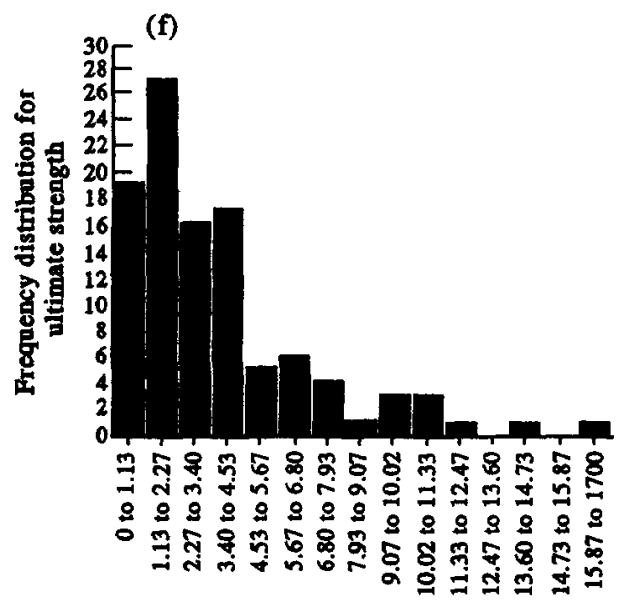

(Units are MPa)

Fig. 2. Distribution plots are shown for: (a) bone volume fraction; (b) trabecular plate number; (c) degree of anisotropy; (d) connectivity (-Euler number normalized by the volume of analyzed); (e) modulus; (f) ultimate strength. These plots of the morphology, anisotropy and mechanical data show a wide range of properties were studied. Only the mechanical properties (e and $f$ ) demonstrated a skewed distribution. 

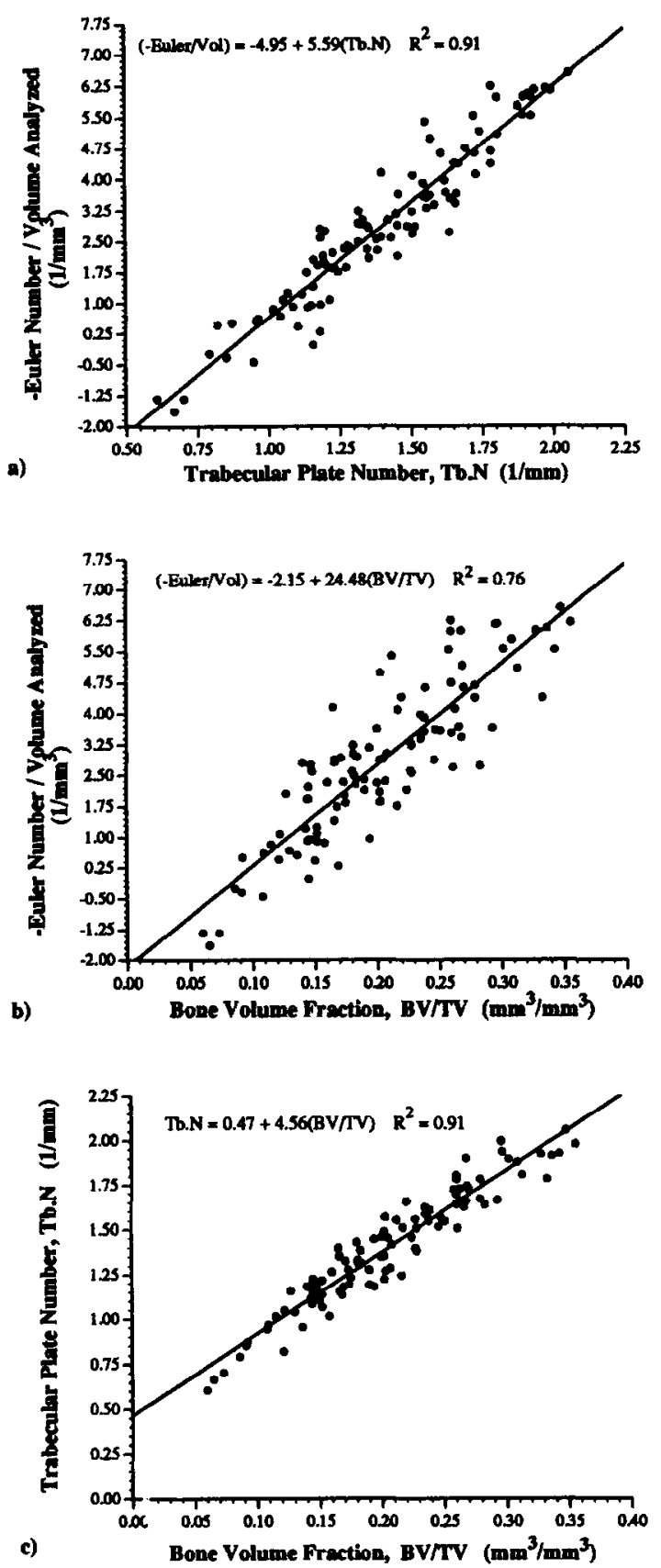

Fig. 3. Plots between some of the morphologic parameters are shown: (a) connectivity vs trabecular plate number; (b) connectivity vs bone volume fraction; (c) trabecular plate number vs bone volume fraction. Strong relationships were found between these independent morphological variables. The linear regression models are included in each graph.

found for the full data set. Thus, all results presented in this paper are for the specimens as originally scanned and thresholded.

The correlations between ash density, apparent density and bone volume fraction are also presented in Table 3. Ash and apparent density are very highly correlated $(r=0.97)$. Bone volume fraction, a stereological measure from micro-CT images, corre-

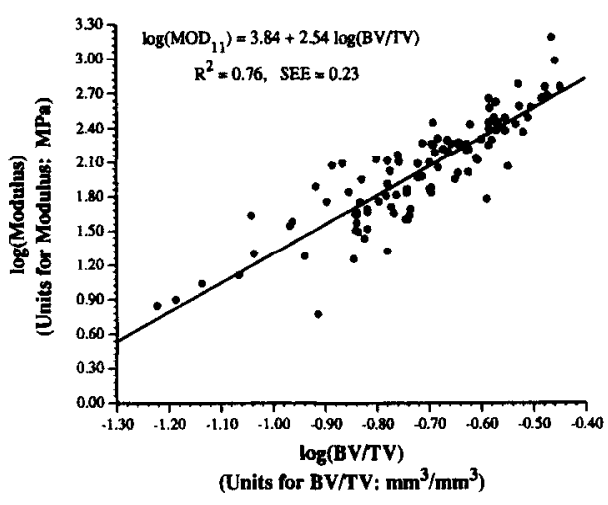

Fig. 4. As can be seen by this plot of AP modulus vs bone volume fraction on the $\log -\log$ scale, strong relationships were found between the mechanical properties and the density of trabecular bone. The regression equation and line are shown with the graph, where ' 11 ' stands for the AP test direction. Regression equations for the ML and IS directions may be found in Table 4 .

lated well with the physical measures of ash density $(r=0.94)$ and apparent density $(r=0.90)$. Because BV/TV was highly correlated with both ash and apparent density as well as to the other morphology variables, only the density measure of BV/TV was included in the regression analyses relating the structure of trabecular bone to its mechanical properties.

The plot of $\log (\mathrm{MOD})$ vs $\log (\mathrm{BV} / \mathrm{TV})$ for the AP test direction is shown in Fig. 4. Similar curves were seen for the ML and IS directions and thus only the AP direction is shown. The log scale is used because a nonlinear relationship was observed between the modulus and bone volume fraction when plotted on the observed scale. The regression equations for each direction and Ave: $\log (M O D)$ are given in Table 4 (first four equations); significant relationships were found with $R^{2}=0.76,0.70$ and 0.69 for each direction, respectively, and $R^{2}=0.88$ for the Ave: $\log (\mathrm{MOD})$ equation. An analysis of the constant and coefficient terms showed that these equations were direction dependent. The coefficients of the equations also depend on the donor and metaphyseal type.

Comparing only the directional measure of the MIL of trabecular bone to the modulus, the plot for the AP test direction data of $\log \left(M O D_{i i}\right)$ vs $\log \left(\mathrm{MIL}_{\mathrm{ii}}\right)$ is shown in Fig. 5 . The regression equations for each direction and an average of $\log$ (MOD) and $\log (\mathrm{MIL})$ are also given in Table 4 (second set of four equations); all equations were significant and $R^{2}=0.70,0.57$ and 0.65 for each direction, respectively, and $R^{2}=0.77$ for the Ave: $\log (M O D)$ vs Ave $: \log (\mathrm{MIL})$ equation. Much of the variance is still unexplained in the modulus using just this directional measurement. The relationship was also dependent on the direction of testing and the dummy variables for donor and metaphyseal type.

A relationship using both global (BV/TV and DA) and directional $\left(\mathrm{MIL}_{\mathrm{ii}}\right)$ measurements was developed to investigate if a single relationship independent of 
Table 4. Regression models for modulus, MOD. The 'Ave' direction equations were found by using the arithmetic mean of $\log \left(\mathrm{MOD}_{\mathrm{ii}}\right)$ and $\log \left(\mathrm{MIL}_{\mathrm{ii}}\right)$. $p$-values $<0.005$ for all coefficients and equations except where denoted by *. Directions $11=\mathrm{AP}, 22=\mathrm{ML}, 33=\mathrm{IS}$, and Ave $=$ (average value of the log of the directional variables from all three directions). The standard error of the estimate, SEE, is given in the last column. $N=104$ for all equations

\begin{tabular}{lcccccc}
\hline $\log \left(\mathrm{MOD}_{\mathrm{ii}}\right)$ & Constant & $\log (\mathrm{BV} / \mathrm{TV})$ & $\log \left(\mathrm{MIL}_{\mathrm{ii}}\right)$ & $\log (\mathrm{DA})$ & $R^{2}$ & SEE \\
\hline 11 & 3.84 & 2.54 & & & 0.76 & 0.23 \\
22 & 3.76 & 2.61 & & & 0.70 & 0.28 \\
33 & 3.80 & 2.10 & & & 0.69 & 0.23 \\
Ave & 3.80 & 2.42 & & & 0.88 & 0.14 \\
11 & 4.52 & & 4.75 & & 0.70 & 0.25 \\
22 & 5.04 & & 5.37 & & 0.57 & 0.33 \\
33 & 4.18 & & 4.19 & & 0.65 & 0.24 \\
Ave & 5.44 & & 6.47 & & 0.77 & 0.20 \\
11 & 4.43 & 1.64 & 2.35 & & 0.84 & 0.19 \\
22 & 5.00 & 1.89 & 3.01 & & 0.82 & 0.21 \\
33 & 4.42 & 1.38 & 2.52 & & 0.84 & 0.16 \\
Ave & 4.34 & 1.88 & 1.79 & & 0.90 & 0.13 \\
11 & 4.55 & 1.69 & 2.36 & $-0.37^{*}$ & 0.84 & 0.19 \\
22 & 4.99 & 2.25 & 2.14 & -1.13 & 0.84 & 0.20 \\
33 & 4.83 & 1.31 & 3.18 & $-0.79 *$ & 0.85 & 0.16 \\
Ave & 4.56 & 1.90 & 1.95 & -0.54 & 0.91 & 0.13 \\
\hline
\end{tabular}

$* p=0.151$ for $11, p=0.011$ for 33 .

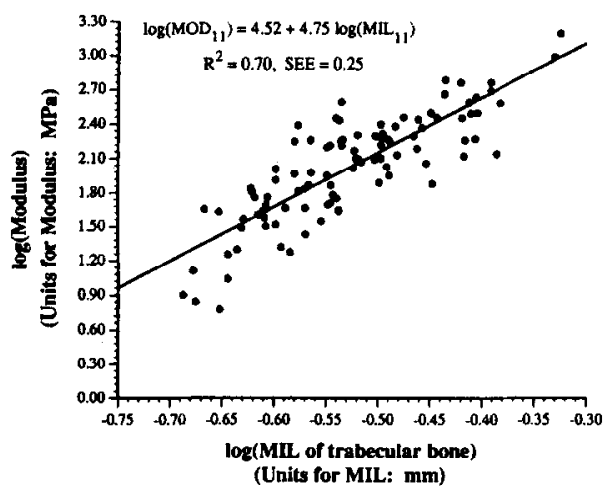

Fig. 5. As can be seen by this plot for the AP direction of modulus vs the mean intercept length of trabecular bone on the log-log scale, strong relationships were found between the mechanical properties and directional measures of trabecular bone. The regression equation and line are shown with the graph, where ' 11 ' stands for the AP test direction. Regression equations for the ML and IS directions may be found in Table 4.

direction of testing could be formulated to estimate $\mathrm{MOD}_{\mathrm{ii}}$. Using just $\log (\mathrm{BV} / \mathrm{TV})$ and $\log \left(\mathrm{MIL}_{\mathrm{ii}}\right)$ in the regression analysis, a significant relationship was found for each direction and for the equation relating Ave: $\log (\mathrm{MOD})$ to Ave: $\log (\mathrm{MIL})$; for the equation using the averages, $R^{2}=0.90$ and SEE $=0.13$ (see Table 4, third set of four equations). Because the constant and coefficient terms were significantly different for each direction and from those from the equation using the average variables, the equations for modulus are dependent on the direction of testing. These equations were also dependent on the dummy variables for donor and metaphyseal bone type. Adding in the DA to the equations (last set of four equations of Table 4) did improve the amount of variance explained slightly (for the Ave relationship $R^{2}=0.91$ ), but the relationships were still dependent on the direction of testing. Metaphyseal type and donor also had a major effect on this relationship.

The other mechanical property measured was ultimate strength, $S_{u}$. The ultimate strength was first compared to the elastic tangent modulus for the direction of failure on a $\log -\log$ scale. A strong relationship was found between the two variables:

$$
\begin{aligned}
\log \left(\mathrm{S}_{\mathrm{ui}}\right) & =-1.26+0.79 \log \left(\mathrm{MOD}_{\mathrm{ii}}\right), \\
R^{2} & =0.95, \quad \mathrm{SEE}=0.08,
\end{aligned}
$$

the relationship did not differ significantly between direction of testing, and metaphyseal type and donor had little effect.

To develop an empirical relationship between the ultimate strength with the morphology and architectural measures similar to that done for the modulus, regression models were also fit using the logarithms of the variables. Using only the bone volume fraction as a predictor for ultimate strength, the relationship for each direction was found to be dependent on the direction of testing, $p<0.0001$ (see Table 5, first set of three equations). The relationship using only the mean intercept length of trabecular bone (Table 5, second set of four equations) was also found to be dependent on the direction of testing $(p<0.002)$. The effect of donor and metaphyseal type on these equations were small.

The variables of BV/TV, MIL $L_{i i}$, and DA were used to develop a relationship for the ultimate strength appropriate for any direction of testing. Using only $\mathrm{BV} / \mathrm{TV}$ and $\mathrm{MIL}_{\mathrm{ii}}$ (Table 5, third set of four equations), a strong relationship was found $\left(R^{2}=0.89\right.$ and 
Table 5. Regression models for ultimate strength, $S_{u}$. p-values $<0.005$ for all coefficients and equations except where denoted by $*$. Directions $11=A P, 22=M L, 33=I S$, and All $=($ all three directions regressed together). For the AP, $\mathrm{ML}$, and IS directions $N=32,34$, and 38 respectively, and $N=104$ for equations of All directions regressed together. The standard error of the estimate, SEE, is given in the last column

\begin{tabular}{lcccccc}
\hline $\log \left(\mathrm{Su}_{\mathrm{ii}}\right)$ & Constant & $\log (\mathrm{BV} / \mathrm{TV})$ & $\log \left(\mathrm{MIL}_{\mathrm{ii}}\right)$ & $\log (\mathrm{DA})$ & $R^{2}$ & $\mathrm{SEE}$ \\
\hline 11 & 2.12 & 2.47 & & & 0.77 & 0.17 \\
22 & 1.85 & 2.26 & & & 0.82 & 0.19 \\
33 & 1.86 & 1.80 & & & 0.82 & 0.15 \\
11 & 2.37 & & 3.87 & & 0.74 & 0.18 \\
22 & 3.22 & & 5.16 & & 0.72 & 0.23 \\
33 & 2.08 & & 3.34 & & 0.56 & 0.23 \\
All & 2.23 & & 2.56 & & 0.66 & 0.23 \\
11 & 2.59 & 1.52 & 2.20 & & 0.89 & 0.12 \\
22 & 2.85 & 1.53 & 2.62 & & 0.92 & 0.13 \\
33 & 2.24 & 1.41 & 1.46 & & 0.88 & 0.12 \\
All & 2.59 & 1.39 & 2.32 & & 0.89 & 0.13 \\
11 & 2.85 & 1.67 & 2.21 & $-0.69^{*}$ & 0.91 & 0.11 \\
22 & 2.81 & 1.66 & 2.27 & $-0.36^{*}$ & 0.92 & 0.13 \\
33 & 2.47 & 1.38 & 1.80 & $-0.42^{*}$ & 0.89 & 0.12 \\
All & 2.79 & 1.44 & 2.41 & -0.58 & 0.91 & 0.12 \\
\hline
\end{tabular}

${ }^{*} p=0.028$ for $11, p=0.422$ for $22, p=0.254$ for 33 .

$\mathrm{SEE}=0.13$ for the regression equation of all three directions together), but was still slightly dependent on the direction of testing $(p=0.021)$. The effects of donor and metaphyseal type were minimal. Including the DA (last set of four equations in Table 5), the fit improved only slightly $\left(R^{2}=0.91, \mathrm{SEE}=0.12\right.$ for the equation of all three directions together) and was not dependent on the direction of testing $(p=0.17)$. Examining the effects of the metaphyseal type and donor source, it was found that neither were as significant for ultimate strength as they were with modulus.

\section{DISCUSSION}

This paper investigated the relationship between the various structural parameters often measured for trabecular bone as well as how these parameters relate to the mechanical properties. Cubes of trabecular bone were taken from various metaphyseal regions of the body and several donors to ensure a wide range of material properties would be studied. The cubes were scanned in a unique micro-CT system to generate three-dimensional digitizations of the cubes upon which the various stereologic algorithms could be run. The specimens were then subjected to uniaxial compression tests and finally the apparent and ash weight densities measured.

High positive correlations were obtained between the number of trabeculae (Tb.N), bone volume fraction (BV/TV), and connectivity (-Euler/Vol). These three variables are measured using different stereological procedures and have no underlying mathematical relationship yet are highly correlated. These correlations were not affected by the limitations of the scanning and thresholding procedure used as seen for the specimens that had only the most con- tinuous structure analyzed. The effect of trabeculae being disconnected by being at the edge of the analysis volume may alter the relationships slightly but should not have a major effect or change the type of correlations seen. Figure 3 demonstrates that as the amount of bone increases, one can expect that the number of trabeculae and connections to increase. There is probably a limit to the number of connections and trabeculae that can be added. If too much bone material is added, the bone would no longer be cancellous bone, but a porous type of cortical bone. It is also possible that the conformation of the trabecular architecture may follow a consistent pattern in which, for trabeculae whose variation in thickness is small, a constant number of connections are prescribed for a given trabecular tissue volume, or number of trabeculae. Cancellous bone may try to preserve its architecture and density within a certain range; and therefore, a certain mechanical environment. Other investigators have noticed a similar relationship between bone volume fraction (or closely related quantities) and trabecular number, but not many studies have looked at the three-dimensional connectivity for trabecular bone. Snyder et al. (1989b) found a linear relationship between trabecular number and density for vertebral bodies (horizontal trabeculae, $R^{2}=0.90$; vertical trabeculae, $R^{2}=0.87$ ). Turner $e$ al. (1990) also found a significant correlation $(r=0.74)$ between areal porosity and average mean intercept length* for bovine distal femoral cancellous bone and human proximal tibiae.

*Turner et al. (1990) defined MIL as $1 / P_{\mathrm{L}}(\Theta)$ which is equivalent to $1 / \mathrm{Tb} . \mathrm{N}(\Theta)$. This term was subsequently normalized by the areal porosity in the derivation of the anisotropy tensor. 
It should also be noted that the relationships developed in this paper were found for individuals with no known bone disorders and with very different ages (although biased towards the older age groups). There may be a given relationship for normal individuals and bones, but various pathological disorders and/or age may change this relationship. The slope and/or $(y$-axis) intercept point of the curves could change for such individuals. If these relationships do change with age, gender, and disease, it may provide insight into why individuals with approximately the same amount of bone can have different properties and susceptibility to fractures. The linear regression relationships between these variables shown in Fig. 3 did not take into account the donor or metaphyseal type. When these effects were included in the regression analyses for these variables, metaphyseal type was found to have a major effect while donor had a minor effect. Although there is a strong relationship between these architectural measures, it appears they are still dependent on location. Each bone may be trying to organize its structure differently based its loading environment.

In work by Fazzalari et al. (1989), the relationship between bone surface to total volume ratio, $S_{\mathrm{v}}$ (which is equivalent to Tb.N as derived in this paper), to the volume fraction, $V_{\mathrm{v}}$ (denoted $\mathrm{BV} / \mathrm{TV}$ in this paper), was investigated to examine the effects on this relationship if different models are assumed for the trabecular bone structure, that is, whether the bone is made up of plates, rods, or spherical voids. The mathematical relationship developed between Tb.N (or $S_{\mathrm{v}}$ ) and BV/TV (or $V_{\mathrm{V}}$ ) was found to be dependent on the type of model assumed for the trabecular bone. Comparing actual experimental data from the femoral head of controls to osteoarthritic patients and the iliac crest of controls to osteoporotic patients, they found the relationship between Tb.N and BV/TV differed between the control and disease state as well as between the metaphyses. Although our data for the relationship between Tb.N and BV/TV showed a dependence on metaphysis type, there were not enough data points in each metaphyseal type to investigate whether one metaphyseal could be better approximated as a plate or rod-like structure. Because the equation for trabecular plate number, Tb.N, assumes a plate-like model for its derivation, this may be part of the reason for the dependence of metaphyseal type in the relationship between Tb.N and BV/TV. Different metaphases may need to be modeled either as completely plate-like, rod-like, or a mixture of rods and plates to derive a more appropriate measure of trabecular number. Schemes of how the body may try to maintain the relationship between trabecular number and bone volume fraction or connectivity could then be modeled to study better the effects of aging and diseases on these relationships.

A change in the relationship between these morphological parameters (trabecular member, bone volume fraction, and connectivity) may provide a basis for predicting various pathological diseases. Immobilization studies, for example, have been used to examine changes seen in trabecular bone as a model for osteoporosis. From studies on dog forelimbs, Jaworksi and Uhthoff (1986) found a decrease in trabecular bone volume with time, but after remobilization, partial to total recovery of bone volume was seen, depending on the age of the animal. Although they did not quantify the number of trabeculae or how connected the trabeculae were, they felt that bone loss and recovery occurred only on the endosteal cortical and trabecular surfaces. Schaffler and Pan (1992) also found a decrease in trabecular bone volume, thickness and eventually trabecular number in an immobilization study using adult female beagles. In a related study, Yoshida et al. (1991) studied the effects of ovariectomy, sciatic neurotomy, hemicordotomy, and their combinations on the trabecular bone of the rat tibia. They found a decrease in percentage bone area and trabecular width due to immobilization from a hemicordotomy, while the ovariectomy caused a decrease in percent bone area and trabecular number but no real change in trabecular width. A combination of both immobilization (by sciatic neurotomy or hemicordotomy) with ovariectomy caused a greater decrease in percent bone area and a decrease in trabecular number and trabecular width, with the hemicordotomy causing a much greater decrease in trabecular width than that seen with the sciatic neurotomy. Work by Mori et al. (1990) found that administration of prostaglandin $E_{2}$ to ovariectomized, osteopenic rats caused an increase in cancellous bone mass and trabecular number.

From these studies, it appears that mechanical influences seem to alter the amount of bone by changing the thickness of the trabeculae, while hormonal and/or chemical influences appear to have a direct affect on the number of trabeculae. Together these two influences, mechanical and hormonal, may lead to the balance seen between the amount of bone, number of trabeculae and number of connections for normal bone, but altering one or both of these influences will lead to alterations in this balance. Trabecular bone may develop a new relationship between these morphological parameters to try to maintain a structure of adequate strength based on the relationship allowed between them at a given mechanical and/or hormonal level. The metaphyseal dependency of the morphological correlations found in this study illustrates this concept and suggests that trabecular bone may optimize its structure to accommodate its specific loading environment. This may be one reason why certain bones (and regions within a bone) may be more plate-like while others have a more rod-like structure.

The other morphological variables (BS/BV, Tb.Th, and $\mathrm{Tb} . \mathrm{Sp}$ ) studied in this paper were also highly correlated (Table 3). However, they were derived from bone volume fraction and trabecular number [see equations (1)-(5)] and their interrelationship is dic- 
tated by their derivation. Since the three basic measurements (BV/TV, Tb.N and - Euler/Vol) are all strongly related as well as BV/TV being highly correlated to apparent and ash weight densities, the empirical relationships developed for the mechanical properties used only the morphological variable of bone volume fraction. Including other variables would not improve the relationships in a useful manner.

In determining the morphological and architectural measures, an accurate means of representing the anisotropy of the entire bone cubes was necessary. The use of surface, central or an average representative plane to form the anisotropy tensor should produce similar tensors if the architecture of the specimen is relatively consistent throughout the specimen. If the architecture varies considerably through the specimen, then the choice of analysis planes will have an effect on the resulting tensor. Since the average plane analysis uses most of the three-dimensional data, a more representative tensor of the specimen will be produced.

In a related study, it was found that for cubes that had two-dimensional MIL ratios (degree of anisotropy) approaching 1.2 on the planes of the analysis, the predicted direction of orientation could vary by $20^{\circ}$ or more (Kuhn, 1987; Kuhn et al., 1988). These types of planes are probably close to being 'isotropic' making it imperative that the degree of anisotropy be examined as well as the predicted directions when trying to relate principal structural directions to principal mechanical properties. As noted by Turner and Cowin (1988), errors in the principal structural directions will lead to larger errors in predicting the mechanical properties. For specimens that are highly anisotropic, the error in predicting the principal structural directions will be small when using surface planes, but that error may increase as the cube becomes more isotropic or the orientation varies greatly while having the same degree of anisotropy. Since there are errors in making the mean intercept measurements as well as in the mechanical properties, efforts should be made to reduce their effect on the analysis. It is partly due to this that only the tensor produced by the average plane analysis was studied.

It should be noted that the formula used for determining the mean intercept length is different from that used by other investigators (Cowin, 1986; Harrigan and Mann, 1984; Turner et al., 1990), but is the same as that originally proposed by Whitehouse (1974) and that used by Hayes and Snyder (1981) and Snyder et al. (1989a). Although mathematically the definitions are not very different, there is an important conccptual distinction. The difference in the formulae has to do with the term $P_{\mathrm{P}}$. Including the factor $P_{\mathrm{P}}$, as in equation (6), gives the mean intercept length of trabecular bone, i.e. the average thickness of bone in the direction that MIL is calculated. With this definition, MIL seems appropriate to be included as an independent term in a structure-function relationship. Leav- ing $P_{p}$ out produces the mean intercept length of one trabeculae/marrow length (i.e. the combined length of one trabeculae and the adjacent marrow cavity in the direction the test lines). However, structures can be constructed with nearly identical $1 / P_{\mathrm{L}}(\mathbf{n})$ yet have vastly different mechanical properties due to different amounts of structural material. In the relationship developed by Turner et al. (1990), they did normalize the mean intercept length they calculated by the areal porosity that was then used to determine the anisotropy tensor. This tensor was converted to the fabric tensor (inverse square root of the anisotropy tensor) and then normalized by its trace. It was these normalized fabric tensor values that were then used to develop the structure-function relationship. Part of the reason for using the normalized fabric tensor was based on the work by Cowin (1985) where he developed an analytical relationship between the compliance matrix with the density and fabric tensor for trabecular bone. To use this tensor relationship, one has to assume at least orthotropic symmetry and to test trabecular bone along the principal structural directions. Since so few of the cubes from this study had a test direction aligned with the principal structural directions as determincd from the anisotropy tensor (only nine cases), we are not able to formulate a relationship similar to that presented by Cowin (1985) or that used by Turner et al. (1990). The relationship we developed was purely empirical and does not use a normalized mean intercept length.*

In addition to the possible errors associated with the stereologic analyses that will cause some of the unexplained variance in the estimation of the mechanical properties, there will also be errors in the mechanical data itself. In this study, specimens where tested unconfined, without a lubricant on the platen heads, and the displacement was measured across the platen heads, not by an extensiometer attached to the central portion of the specimen. All of these factors can lead to errors in the estimation of what the true mechanical properties may be for each specimen (Allard and Ashman, 1991; Linde and Hvid, 1989; Odgaard and Linde, 1991), but this error should be systematic for all specimens. Although we do not know precisely the effects of the end constraints on our specimens or precisely where they failed, our data are consistent with previously published studies, as reflected in the relationship between strength and modulus. Review of all the load-deformation curves demonstrated a pattern of failure not consistent with isolated 'edge' failure. While, as with most studies of trabecular bone, these data cannot be considered as true mechanical propertics (due to nonphysiologic boundary conditions), all specimens were tested under the same protocol and the critical results are only dependent on the

*An empirical relationship using MIL divided by the trace of the MIL tensor as well as BV/TV and DA for estimating the modulus or ultimate strength explained about the same amount of variance as did using just MIL. 
contrasts among the specimens, reflective of architectural variations.

The results from the mechanical tests showed strong correlations between the morphology and modulus values (Table 4). The regression analysis consisted of using transformed values for both the mechanical properties, as the dependent variables, and the morphology and architectural measures, as the independent variables. Because the relationships were not linear and the variance in the data became greater for larger values, the analysis was performed on the log scale to try to minimize the effect of the variance. Although the SEE may seem large when converted back to the original scale, this error in the predicted modulus represents a small percentage of its range.

Bone volume fraction and the mean intercept length of trabecular bone were found to be good predictors for the mechanical properties. When either variable was used alone as a single estimator, the direction of testing was required in the model. Even though the mean intercept length is a directional measurement, used alone it cannot predict the mechanical properties of trabecular bone unless a dummy variable for the direction of testing is included. When using bone volume fraction, the mean intercept length, and degree of anisotropy to estimate the modulus, the relationship was found to be statistically dependent on the direction of testing as well as the metaphyseal type and donor. For the ultimate strength, the relationship was not as dependent on the direction of testing, and only slightly on metaphyseal type and donor. This difference in dependence on direction of testing and specimen location between modulus and ultimate strength is not easily understood. Because modulus is dependent on the direction of testing and specimen location, other features of the architectural arrangement besides the density and amount of bone in the direction of testing may be needed if one is interested in developing a general relationship to predict the modulus independent of direction of testing. For example, using a different procedure to quantify the anisotropy of the trabecular bone, such as proposed by Odgaard et al. (1990), or modeling the trabeculae as a rod-like structure instead of plate-like may be needed. If the goal is to investigate the effects of changing the amount of bone, number of trabeculae, or mean length of the trabeculae, then the relationships developed here and by other investigators are more than adequate. Since modulus is a measure of the slope between stress and strain, while ultimate strength is a measure of the maximum stress the bone can achieve, it seems that modulus would be more dependent on the arrangement and tissue properties than ultimate strength. The dependence of ultimate strength on the properties of the individual trabeculae appears not to be as great, since relationships using the overall bone volume fraction combined with the amount of bone in the direction of testing were found to be independent of the test direction and only slightly dependent on the metaphyseal type and donor.

The variation in mechanical properties due to donor may be due to either age or gender effects, both of which may be important factors in predicting the stiffness of cancellous bone. Other investigators have tried to form general structure function relationships, but often have used specimens from one or two metaphyseal regions, and a limited age range (Snyder $e t$ al., 1989a; Turner et al., 1990). It is known that certain structural properties vary with age (Atkinson, 1967; Bergot et al., 1988; Merz and Schenk, 1970a, b), but how these changes with age affect the mechanical properties is not known. If the gender and age of the donor are important, then the properties of the trabeculae tissue itself may be as important in predicting the mechanical properties of cancellous bone as is the morphology and architectural organization of the trabecular bone. This next level of structural organization in the hierarchy will need investigation to gain a full understanding of the relationship between structural and mechanical properties.

In conclusion, strong correlations were found between the independent structural measures of bone volume fraction, trabecular plate number and connectivity; bone volume fraction was also found to be highly correlated with apparent density and ash weight density. Because of these strong correlations and the dependence of the other morphology measures determined with BV/TV and Tb.N, only bone volume fraction and the mean intercept length of trabecular bone where included in the regression equations to estimate the mechanical properties. Strong relationships were found for modulus and ultimate strength capable of estimating approximately $90 \%$ of the variance in these mechanical properties. The modulus was found to slightly dependent on the direction of testing, and both modulus and ultimate strength were dependent on the metaphyseal type and donor the specimens came from.

Acknowledgements-This work was supported by grants from the National Institutes of Health (AR34399 and AR20557). The authors also wish to thank Anita Grierson, B. S., Laurie Huston, M. S., David Kruger, M. D., Doris McCubbrey, M. S., Sarafin Samson, M. S. and David Viviano, M. S. for help in various aspects of the data collection.

\section{REFERENCES}

Allard, R. N. and Ashman, R. B. (1991) A comparison between cancellous bone compressive moduli determined from surface strain and total specimen deflection. Trans. 37th Ann. Meeting Orthop. Res. Soc. 16, 151.

Atkinson, P. J. (1967) Variation in trabecular structure of vertebrae with age. Calcif. Tiss. Res. 1, 24-32.

Bergot, C., Laval-Jeantet, A. M., Préteux, F. and Meunier, A. (1988) Measurement of anisotropic vertebral bone loss during aging by quantitative image analysis. Calcif. Tiss. Int. 43, 143-149.

Brown, T. D. and Ferguson, A. B. (1980) Mechanical property distributions in the cancellous bone of the human proximal femur. Acta Orthop. Scand. 51, 429-437. 
Carter, D. R. and Hayes, W. C. (1977) The compressive behevior of bone as a two-phase porous structure. J. Bone Jt Surg. 59A, 954-962.

Ciarelli, M. J., Goldstein, S. A., Kuhn, J. L., Cody, D. D. and Brown, M. B. (1991) Evaluation of orthogonal mechanical properties and density of human trabecular bone from the major metaphyseal regions with materials testing and computed tomography. J. orthop. Res. 9, 674-682.

Cowin, S. C. (1985) The relationship between the elasticity tensor and the fabric tensor. Mech. Mat. 4, 137-147.

Cowin, S. C. (1986) Wolff's law of trabecular architecture at remodeling equilibrium. J. biomed. Engng 108, 83-88.

Fazzalari, N. L., Crisp, D. J. and Vernon-Roberts, B. (1989) Mathematical modelling of trabecular bone structure: the evaluation of analytical and quantified surface to volume relationships in the femoral head and iliac crest. $J$. Biomechanics 22, 901-910.

Feldkamp, L. A., Davis, L. C. and Kress, J. W. (1984) Practical cone-beam algorithm. J. Opt. Soc. A1, 612-619.

Feldkamp, L. A., Goldstein, S. A., Parfitt, A. M., Jesion, G. and Kleerekoper, M. (1989) The direct examination of three dimensional bone architecture in vitro by computed tomography. J. Bone Min. Res. 4, 3-11.

Gibson, L. J. (1985) The mechanical behavior of cancellous bone. J. Biomechanics 18, 317-328.

Goldstein, S. A. (1987) The mechanical properties of trabecular bone: dependence on anatomical location and function. J. Biomechanics 20, 1055-1061.

Goulet, R. W., Feldkamp, L. A., Kubinski, D. J. and Goldstein, S. A. (1989) Predicting the architectural orientation of trabecular bone. Trans. 35th Ann. Meeting Orthop. Res. Soc. 14, 263.

Harrigan, T. P. and Mann, R. W. (1984) Characterization of microstructural anisotropy in orthotropic materials using a second rank tensor. J. Mat. Sci. 19, 761-767.

Hayes, W. C. and Snyder, B. (1981) Toward a quantitative formulation of Wolff's law in trabecular bone. In Mechanic Properties of Bone (Edited by Cowin, S. C.), pp. 43-68. ASME, Boulder, CO.

Hvid, I., Jensen, N. C., Bunger, C., Solund, K. and Djurhuus, J. C. (1985) Bone mineral assay: its relation to the mechanical strength of cancellous bone. Engng Med. 4, 79-83.

Jaworski, Z. F. G. and Uhthoff, H. K. (1986) Reveribility of nontraumatic disuse osteoporosis during its active phase. Bone 7, 431-439.

Kuhn, J. L. (1987) Trabecular bone structure and mechanical properties. Ph.D. dissertation, University of Michigan, Ann Arbor, MI

Kuhn, J. L., Goldstein, S. A., Feldkamp, L. A., Goulet, R. W. and Jesion, G. (1990) Evaluation of a microcomputed tomography system to study trabecular bone structure. J. orthop. Res. 8, 833-842.

Kuhn, J. L., Goulet, R. W., Goldstein, S. A. and Feldkamp, L. A. (1988) A study of variation of trabecular architectures in small volumes of bone using a microcomputed tomography system. Proc. 12th American Soc. of Biomechanics Meeting, Urbana-Champaign, IL, pp. 16-17. ASB.

Linde, F. and Hvid, I. (1987) Stiffness behavior of trabecular bone specimens. J. Biomechanics 20, 83-89.

Linde, F. and Hvid, I. (1989) The effect of constraint on the mechanical behaviour of trabecular bone specimens. J. Biomechanics 22, 485-490.

Merz, W. A. and Schenk, R. K. (1970a) Quantitative structural analysis of human cancellous bone. Acta Anat. 75, 54-66.

Merz, W. A. and Schenk, R. K. (1970b) A quantitative histological study of bone formation in human cancellous bone. Acta Anat. 76, 1-15.

Mori, S., Jee, W. S. S., Li, X. J., Chan, S. and Kimmel, D. B. (1990) Effects of prostaglandin $E_{2}$ on production of new cancellous bone in the axial skeleton of ovariectomized rats. Bone 11, 103-113.

Odgaard, A., Jensen, E. B. and Gundersen, H. J. G. (1990)
Estimation of structural anisotropy based on volume orientation. A new concept. J. Microscopy 157, 149-162.

Odgaard, A. and Linde, F. (1991) The underestimation of Young's modulus in compressive testing of cancellous bone specimens. J. Biomechanics 24, 691-698.

Parfitt, A. M., Drezner, M. K., Glorieux, F. H., Kanis, J. A., Malluche, H., Meunier, P. J., Ott, S. M. and Recker, R. R. (1987) Bone histomorphometry: standardization of nomenclature, symbols, and units, $J$. Bone Min. Res. 2, 595-610.

Parfitt, A. M, Mathews, C. H. E, Villanueva, A. R., Kleerekoper, M., Frame, B and Rao, D. S. (1983) Relationships between surface, volume and thickness of iliac trabecular bone in aging and in nsteoporosis. J. clin. Invest. 72, 1396-1409.

Pugh, J. W., Rose, R. M. and Radin, E. L. (1973) Elastic and viscoelastic properties of trabecular bone: dependence on structure. J. Biomechanics 6, 475-485.

Raux, P., Townsend, P. R., Miegel, R., Rose, R. M. and Radin, E. L. (1975) Trabecular architecture of the human patella. J. Biomechanics 8, 1-7.

Rice, J. C., Cowin, S. C. and Bowman, J. A. (1988) On the dependence of elasticity and strength of cancellous bone on apparent density. J. Biomechanics 21, 155-168.

Schaffler, M. B. and Pan, H. Q. (1992) Alterations of trabecular microarchitecture during immobilization-induced bone loss. Trans. 38th Ann. Meeting Orthop. Res. Soc. 17, 239.

Serra, J. (1982) Image Analysis and Mathematical Morphology. Academic Press, London.

Singh, I. (1978) The architecture of cancellous bone. J. Anat. 127, 305-310.

Snyder, B. D., Cheal, E. J., Hipp, J. A. and Hayes, W. C. (1989a) Anisotropic structure-property relation for trabecular bone. Trans. 35th Ann. Meeting Orthop. Res. Soc. $14,265$.

Snyder, B. D., Edwards, W. T., Van der Linde, J. M., White III, A. A. and Hayes, W. C. (1989b) Stereologic assessment of trabecular structure in the lumbar vertebral body: biomechanical implications. Trans. 35th Ann. Meeting Orthop. Res. Soc. 14, 262.

Townsend, P. R., Raux, P., Rosie, R. M., Miegel, R. E. and Radin, E. L. (1975) The distribution and anisotropy of the stiffness of cancellous bone in the human patella. J. Biomechanics 8, 363-367.

Turner, C. H. (1986) Boundary length and internal surface area measurements in porous materials with elliptical pores. J. Mat. Sci. 21, 3907-3911.

Turner, C. H. and Cowin, S. C. (1988) Errors induced by off-axis measurements of the elastic properties of bone. J. biomed. Engng 110, 213-215.

Turner, C. H., Cowin, S. C., Rho, J. Y., Ashman, R. B., Rice, J. C. (1990) The fabric dependence of the orthotropic elastic constants of cancellous bone. J. Biomechanics 23, 549-561.

Underwood, E. E. (1970) Quantitative Stereology. AddisonWesley, Reading, MA.

Weibel, E. R. (1979) Stereological Methods, Vol 1: Practical Methods for Biological Morphometry. Academic Press, London.

Weibel, E. R. (1980) Stereological Methods, Vol 2: Theoretical Foundations. Academic Press, London.

Whitehouse, W. J. (1974) The quantitative morphology of anisotropic trabecular bone. J. Microscopy 101, 153-168.

Whitehouse, W. J., Dyson, E. D. and Jackson, C. K. (1971) The scanning electron microscope in studies of trabecular bone from a human vertebral body. J. Anat. 108, 481-496.

Williams, J. L. and Lewis, J. L. (1982) Properties and an anisotropic model of cancellous bone from the proximal tibial epiphysis. J. biomed. Engng 104, 50-56.

Yoshida, S., Yamamuro, T., Okumura, $H$. and Takahashi, $H$. (1991) Microstructural changes of osteopenic trabeculae in the rat. Bone 12, 185-194. 